\title{
The relative stabilities of the reverse and normal polarity states of the earth's magnetic field
}

\author{
P.L. McFadden ${ }^{1}$, R.T. Merrill ${ }^{2}$, William Lowrie ${ }^{3}$ and Dennis V. Kent ${ }^{4}$ \\ ${ }^{\prime}$ Division of Geophysics, Bureau of Mineral Resources, G.P.O. Box 378, Canberra, A.C.T. 2601 (Australia) \\ 2 Geophysics Program, University of Washington, Seattle, WA 98195 (U.S.A.) \\ ${ }^{3}$ Institut für Geophysik, E.T.H.-Hönggerberg, CH-8093 Zürich (Switzerland) \\ ${ }^{4}$ Lamont-Doherty Geological Observatory, and Department of Geological Sciences, Palisades, NY I0964 (U.S.A.)
}

Received August 22, 1986; revised version received December 16, 1986

\begin{abstract}
Recent analyses of the geomagnetic reversal sequence have led to different conclusions regarding the important question of whether there is a discernible difference between the properties of the two polarity states. The main differences between the two most recent studies are the statistical analyses and the possibility of an additional 57 reversal events in the Cenozoic. These additional events occur predominantly during reverse polarity time. but it is unlikely that all of them represent true reversal events. Nevertheless the question of the relative stabilities of the polarity states is examined in detail, both for the case when all 57 "events" are included in the reversal chronology and when they are all excluded. It is found that there is not a discernible difference between the stabilities of the two polarity states in either case. Inclusion of these short events does, however, change the structure of the non-stationarity in reversal rate, but still allows a smooth non-stationarity. Only 7 of the 57 short events are pre-38 Ma, but the evidence suggests that this is a real geomagnetic phenomenon rather than degradation of the magnetic recording or a bias in observation. This could be tested by detailed magnetostratigraphic and oceanic magnetic surveys of the Paleogene and Late Cretaceous. Overall it would appear that the present geomagnetic polarity timescale for $0-160 \mathrm{Ma}$ is probably a very good representation of the actual history, and that different timescales and additional events now represent only changes in detail.
\end{abstract}

\section{Introduction}

The magnetic field reversal chronology represents one of the best long-term records of the earth's magnetic field in which to search for differences between the properties of the two polarity states. It may also be used to determine if there have been any long-term variations in some overall properties of the field. Recently, both Lowrie and Kent [1] (referred to hereafter as L \& K) and McFadden and Merrill [2] (referred to hereafter as M \& M) have analysed geomagnetic polarity timescales for the Cenozoic and Late Mesozoic. They independently concluded that the statistical properties of the reversal sequence have been non-stationary. A clear $10^{8}$-year variation is present in this record and $M \& M$ suggest this variation is associated with changes in the temperature at the core-mantle interface. However, there were disagreements on other points, including the important question as to whether there is a discerni- ble difference between the properties of the two polarity states. If there is a difference between the two states then this must be attributed to boundary or initial conditions, because the governing equations for the geodynamic indicate that they should (statistically speaking) be identical except for sign [3].

The reasons for the differences in interpretation involve both the statistical procedures and the data sets used. L \& $\mathrm{K}$ analysed the LaBrecque, Kent and Cande [4] scale (referred to hereafter as LKC) using estimation techniques suggested by Naidu [5], while $M \& M$ analysed the Ness, Levi and Couch [6] scale (referred to hereafter as NLC) using the statistical methods of McFadden [7]. $M \& M$ showed that there are instabilities in the "conventional" statistical approach in that insertion of one or two short reversal events (or chrons) can lead to large changes in estimates of the statistical parameters, and a lack of appreciation of this sensitivity has led others to the conclu- 
sion that there are significant differences between the two polarity states. Consequently $\mathrm{M} \& \mathrm{M}$ used the statistical procedures developed by $\mathrm{McF}$ adden [7] that take into account the likelihood that the known reversal chronology is incomplete. They argued that it would take a very "unusual" distribution of missed polarity events to invalidate their conclusion that there is no discernible difference in stability between the two polarity states, a conclusion at variance with previous analyses on the subject (e.g. $[1,8]$ ). However, L \& $\mathrm{K}$ and Kent [9] point out that there is some evidence for 57 reversal events in the Cenozoic that are not included in most reversal chronologies, including the one analysed by $\mathrm{M} \& \mathrm{M}$. Moreover, 46 of these events exhibit normal polarity, while only 11 exhibit reverse polarity.

In this paper we briefly review the evidence for these possible reversal events and consider the apparent disproportionate distribution in favour of normal polarity. We then investigate the effect of including these events into the reversal chronology record and consider the implications from the point of view of core processes and the presently available chronologies.

\section{Evidence for missed short polarity events}

Blakely and Cox [10] and Blakely [11] used a stacking procedure to enhance marine magnetic profiles, and identified several previously unrecognised short-wavelength anomalies that they interpreted as reversal events. Some of the amplitudes of these anomalies appear comparable in size to the M0 to M25 magnetic anomalies that precede the long Cretaceous Normal Polarity Interval, and which are generally interpreted as recording reversal events. Certainly, from a physical point of view, it would not be at all surprising if many short events have been missed, and the statistical properties of the timescales themselves indicate that this is the case. This problem was recognised early on by Cox [12] and Harrison [13]. M \& M predicted that about $46 \%$ of the intervals in the NLC scale contained a "missed" very short event-i.e. on the order of 90 short events missed (though it should be recognised that this estimate is imprecise).

Although the above seems to argue for the interpretation of the "tiny wiggles" (as L \& K called the "new" anomalies that were resolved by stacking the marine magnetic profile records) as short reversal events, there are also reasons for doubting this interpretation. These tiny wiggles reflect changes in intensity of the magnetic field as sensed at the sea surface. Such changes can occur because of (1) true reversal events, (2) magnetic excursions, (3) changes in intensity of the earth's magnetic field not associated with reversals or excursions [14], and (4) changes in rock magnetic properties (including the thickness of the anomaly-producing layer). Distinguishing between these possibilities is difficult, particularly considering that there is noise present and there are other complexities in the recording system $[15,16]$.

There is the possibility of a secondary magnetization, such as a viscous remanent magnetization (VRM), being responsible for many of the tiny wiggles. Naturally if the magnetic mineralogy were uniform throughout the oceanic crust a VRM would only shift the baseline of the anomaly. However, because the mineralogy (including grain size) will vary, some parts of the crust will acquire a VRM more easily than others. Thus it is conceivable that Brunhes VRM overprints could produce apparent normal events during reverse polarity times. This is an attractive hypothesis because it would help explain why the overwhelming majority of the tiny wiggles have been identified as having normal polarity. However, both this and other rock magnetic possibilities can be largely discounted. The tiny wiggles occurred at the same time at very different geographical locations, and on segments of plates with different spreading rates (e.g. northeast Pacific, East Pacific Rise, and the Indian-Antarctic Ridge [11]). The titanomagnetite grains, which predominantly carry the remanence in the rocks producing these anomalies, were some of the last grains to crystallise out from a magma produced by the partial melting of upper mantle material. Subsequent chemical alteration of some of these titanomagnetite grains appears to have had a significant effect upon the magnitude of these anomalies (e.g. see review by Johnson [17]). Under such circumstances it is difficult to conceive how an anomaly associated with variations in rock magnetic properties in one location could be correlated in time with an anomaly associated with rock magnetic properties in a location thousands of kilometers away and on a different 
plate. However, it is easily conceivable that a normal polarity VRM associated with the present normal (Brunhes) epoch could make it difficult, and sometimes impossible, to detect short reverse events. We conclude that few, if any, of the tiny wiggles identified by Blakely and Cox [10] and Blakely [11] originate because of rock magnetic variations. However, the disproportionately larger number of short "normal polarity" anomalies may well be a result of the masking of short "reverse polarity" anomalies by secondary components of magnetization.

At least some of the tiny wiggles probably originate by mechanisms (2) or (3) given above, and it is conceivable the vast majority may do so. Excursions of the field are likely and at least some mechanisms of excursions produce significant changes in the global intensity [18]. As discussed by McFadden [7] the analysis used takes into account both reversal events that are missed and unsuccessful reversals. If reversals, unsuccessful reversals and excursions represent a continuum of behaviour and are all triggered by the same process, it is reasonable to include observed excursions within the reversal statistics. Excursions aside, the dipole field appears to have undergone large changes in intensity even during the Holocene: the dipole field appears to have varied by as much as $30-40 \%$ of its present value $[19,20]$. There is now some evidence that significant changes in intensity can last for periods at least up to a few tens of thousands of years, and probably longer $[18,20]$. It is to be expected that changes such as these would show up on high-resolution magnetic anomaly analyses like those made by Blakely and Cox [10] and Blakely [11]. Indeed, it was reasoning similar to this that led LaBrecque et al. [4] to omit most of the tiny wiggles from their tabulated list of polarity interval ages (see also Cande and LaBrecque [14]).

Overall, therefore, it seems likely that there is more than one mechanism operating to produce the tiny wiggles observed in marine magnetic anomalies. Some of these probably do represent previously missed reversal events, as predicted by M \& M, but others probably do not. More highquality magnetostratigraphic work on terrestrial rocks seems required to determine the proportion of these tiny wiggles that truly represent reversal events.
Clearly it is not possible at this stage to distinguish between those wiggles that do represent a missed event and those that do not, so in the remainder of this paper we simply assume that all 57 of the tiny wiggles included by $\mathrm{L} \& \mathrm{~K}$ do represent short reversal events. Because a disproportionate number (46) of these have been identified as being of normal polarity, this should provide a severe test of the robustness claimed by $M \& M$ and McFadden [7] for their analysis. If the major statistical characteristics of the reversal chronology remain essentially unchanged after including these "events", then one should have considerable confidence that those characteristics have been properly delineated.

\section{The gamma distribution}

As shown in the previous analyses, a gamma distribution provides a good description for the distribution of the intervals, $x$, between observed reversals. Thus if $P(x) \mathrm{d} x$ is the probability of observing an interval in the range $x$ to $(x+\mathrm{d} x)$ then:

$P(x) \mathrm{d} x=\frac{1}{\Gamma(k)}(k \lambda)^{k} x^{k-1} \exp (-k \lambda x) \mathrm{d} x$

where $\Gamma(k)$ is the gamma function of $k$. The mean length $\mu$ and the variance $\operatorname{Var}(x)$ of the intervals are given by:

$\mu=\langle x\rangle=(1 / \lambda)$

$\operatorname{Var}(x)=\mu^{2} / k=1 / k \lambda^{2}$

For an explanation of the physical interpretation of the parameter $k$, see McFadden [7], M \& M and McFadden \& Merrill [21]).

\section{Estimates for $k$}

4.1. Analysis without the additional short events

Estimates for $k$ for the LKC timescale are plotted in Fig. 1. The sequence was analysed using a sliding window that included 25 intervals of each polarity, with the window shifting by one interval of each polarity for each estimate. McFadden [7] has discussed the problems arising from the use of such sliding windows, particularly the visual effect of there being far more informa- 


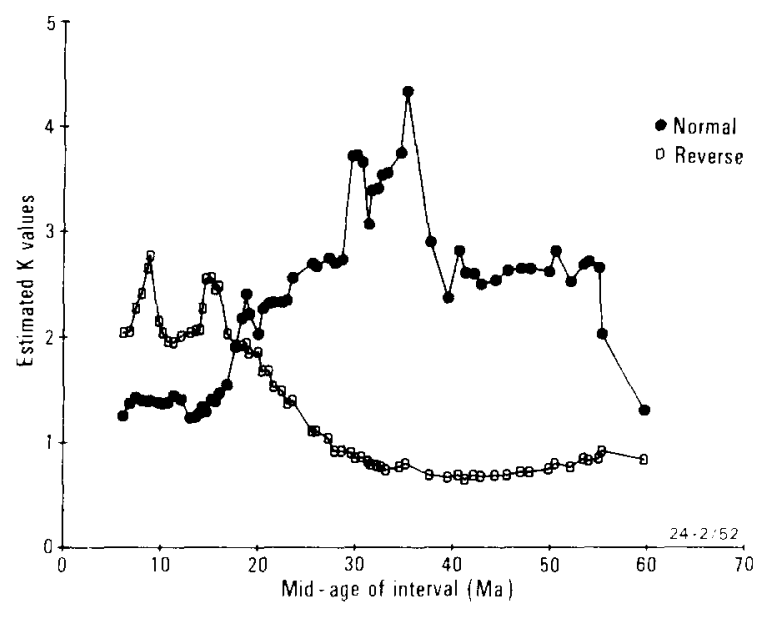

Fig. 1. Estimates of $k$ for both polarities for the LKC timescale. Sliding window covers 25 intervals of each polarity and shifts by one interval of each polarity each time.

tion than is actually available, and the apparent differences produced by the fact that sliding windows provide a concerted search for the maximum difference between estimates. However, if these problems are borne in mind, sliding windows do provide a useful method of analysis.

The large peak in the estimated $k$ for the normal polarity sequence gives the visual impression of a significant difference between $k$ for the normal polarity and $k$ for the reverse polarity. Using the methods of McFadden [7] it may be shown that there is in fact no reason to reject the hypothesis that the normal and reverse polarity sequences share a common $k$, but that the apparent visual difference is due to the extreme sensitivity of the estimation procedure for $k$ and the use of sliding windows. (Reference should be made to McFadden [7] and $M \& M$ for a full explanation and discussion of the problems associated with the analysis.) Unfortunately, such arguments, although entirely valid, are unlikely to be intuitively convincing when faced with a plot such as in Fig. 1. To overcome this problem a single short normal interval has been added into the sequence from $34.00 \mathrm{Ma}$ to $34.01 \mathrm{Ma}$, and the estimates for $k$ from the resulting sequence are plotted in Fig. 2. The fact that the addition of a single interval, into a sequence with a total of 176 intervals, can produce such a large change, is a clear indication of the sensitivity of the analysis.

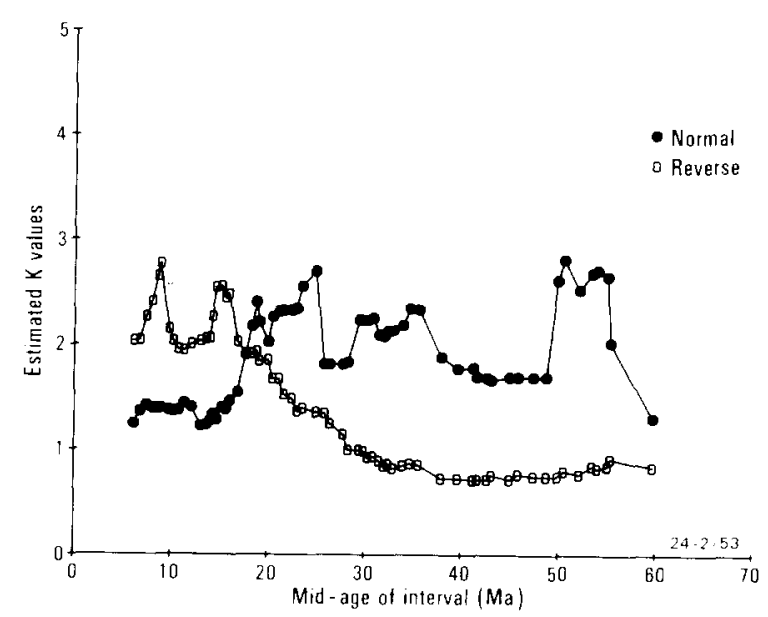

Fig. 2. Estimates of $k$ for both polarities for the LKC timescale with one short interval added at 34.00 to $34.01 \mathrm{Ma}$. Sliding window as for Fig. 1.

Certainly the estimates plotted in Fig. 2 give no reason to reject the hypothesis of a common $k$ for the reverse and normal polarity sequences.

Figs. 1 and 2 here should be compared with Figs. $2 b$ and 5 of $M \& M$ to see how strikingly similar are the statistical properties of the NLC and LKC timescales.

\subsection{Analysis with the additional short events}

The durations of the short events cannot be determined from the marine records, and so values have to be determined in a fairly arbitrary manner. L \& K chose to overcome this problem by simply assigning equal durations to each of the events. On the basis that it was unlikely the durations were longer than about $40 \mathrm{kyr}$, they chose to investigate the effects of setting this duration at 20,30 or $40 \mathrm{kyr}$. This choice of a single duration might initially appear unrealistic. However, experimentation shows that it is in fact a satisfactory approach. Furthermore, it is of little consequence which of 20,30 or $40 \mathrm{kyr}$ is chosen as the arbitrary duration. For simplicity the results and discussion presented here relate to inclusion of the 57 events with a duration of $20 \mathrm{kyr}$, but the conclusions are the same for durations of 30 or 40 kyr.

Before looking at the analysis it is interesting to consider the prediction made by $M \& M$ that about $46 \%$ of the observed intervals contained a 
"missed" short interval. For the LKC scale that would imply about 40 short intervals missed for each of the normal and reverse polarities. Of the 57 short events included by L \& K, 46 are normal and 11 reverse. This would appear to be about the right number of normal intervals, but not enough reverse intervals. Consequently we should expect that inclusion of these short events would stabilise $k$ for the normal sequence at about 1 .

The estimated values of $k$ are plotted in Fig. 3, and as is to be expected, the results appear quite different from Fig. 1. The estimated values for $k$ for the normal polarity do appear to have been stabilised at about 1, as was suggested in the previous paragraph should be the case. In contrast, the estimated values for the reverse polarity sequence have been destabilised and now are typically larger than for the normal polarity sequence. It was this that led L \& $\mathrm{K}$ to state that "The added short events have altered the sense of the asymmetry between the normal and reversed states". However, even with the very sharp peak plotting at $3.5 \mathrm{Ma}$ (the mid-point of the interval 0.11-6.97 Ma, which includes 25 intervals of each polarity), the results do not in fact give good reason for rejecting the hypothesis of a common $k$ for the normal and reverse sequences.

Even though the data give no reason for rejecting the hypothesis of a common $k$, it is important to understand what happens to the sequences when a short polarity chron is added. When a short

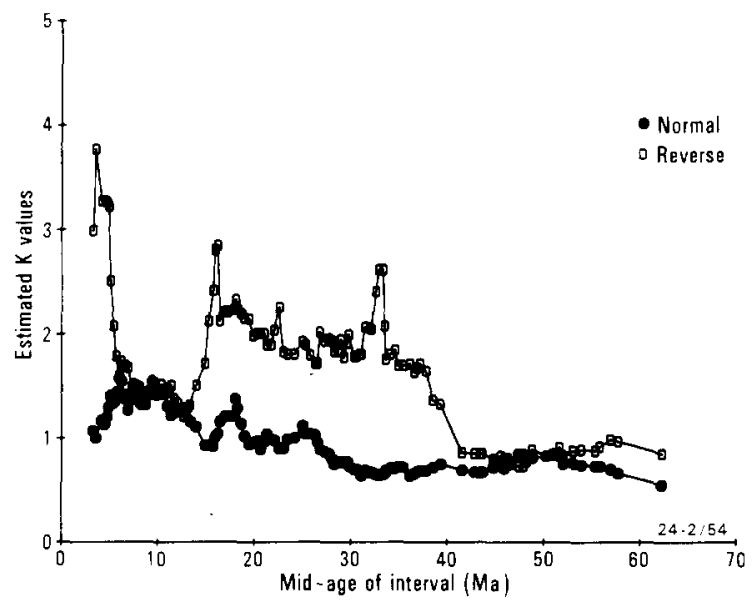

Fig. 3. Estimates of $k$ for both polarities for the LKC timescale with the 57 short intervals added, each with a duration of $20 \mathrm{kyr}$. Sliding window as for Fig. 1. normal polarity chron is added it must, of course, be added into what was previously a reverse polarity chron. Naturally, its position within this reverse polarity chron does not in any way affect the statistical properties of the new normal polarity sequence, only its duration has an effect. In contrast, in the new reverse polarity sequence, a single chron is replaced by two shorter chrons. Because the inserted normal polarity chron is very short, its chosen duration has little effect on the durations of the two new reverse polarity chrons, but its positioning within the original reverse polarity chron determines almost entirely the durations of the two new reverse polarity chrons. Thus, since most of the added chrons are of normal polarity, we are in the rather strange situation where the statistical properties of the new normal polarity sequence are determined by the fact that the added chrons are very short, whereas the statistical properties of the reverse polarity sequence are determined by the positioning of the very same chrons.

Clearly the mean length, $\mu$, of intervals in the new reverse polarity sequence is not affected by variations in the positioning of the short normal polarity chrons, but the variance of reverse polarity interval lengths is. Thus, as may be seen from equation (3), the value of $k$ for the new reverse polarity sequence is strongly affected by the positioning of the short normal polarity chrons. If a set of new normal polarity chrons is spaced uniformly within what was a single reverse polarity chron, then the variance of interval lengths in the new reverse sequence is reduced, and this will appear as an increased estimate for $k$.

Unfortunately, when splitting up one fairly short interval into several smaller intervals (by the insertion of very short intervals of the opposite polarity) there is a natural tendency to space the inserted intervals so that they split the original interval into approximately equal lengths. That this has happened may be seen very clearly from Table 1, which shows the original and altered polarity sequences. The original reverse polarity interval 0.95-1.62 $\mathrm{Ma}$ (duration $0.67 \mathrm{Ma}$ ) has been split into 7 new intervals with the durations of the new reverse polarity intervals being 0.13 , $0.13,0.18$ and $0.17 \mathrm{Ma}$. The very next interval in the original reverse sequence, $1.83-2.41 \mathrm{Ma}$ (duration $0.58 \mathrm{Ma}$ ), has been split into five new inter- 
TABLE 1

Polarity intervals for the last $4 \mathrm{Ma}$ with and without the added short polarity chrons (unit is Ma)

\begin{tabular}{|c|c|c|c|c|c|c|c|}
\hline \multicolumn{4}{|c|}{ Original polarity sequence } & \multicolumn{4}{|c|}{ Altered polarity sequence } \\
\hline \multicolumn{2}{|c|}{ normal polarity } & \multicolumn{2}{|c|}{ reverse polarity } & \multicolumn{2}{|c|}{ normal polarity } & \multicolumn{2}{|c|}{ reverse polarity } \\
\hline interval & length & interval & length & interval & length & interval & length \\
\hline \multirow[t]{3}{*}{$0.00-0.70$} & 0.70 & & & $0.00-0.09$ & 0.09 & $0.09-0.11$ & 0.02 \\
\hline & & & & $0.11-0.70$ & 0.59 & & \\
\hline & & $0.70-0.89$ & 0.19 & & & $0.70-0.89$ & 0.19 \\
\hline \multirow[t]{5}{*}{$0.89-0.95$} & 0.06 & & & $0.89-0.95$ & 0.06 & & \\
\hline & & $0.92-1.62$ & 0.67 & & & $0.95-1.08$ & 0.13 \\
\hline & & & & $1.08-1.10$ & 0.02 & $1.10-1.23$ & 0.13 \\
\hline & & & & $1.23-1.25$ & 0.02 & $1.25-1.43$ & 0.18 \\
\hline & & & & $1.43-1.45$ & 0.02 & $1.45-1.62$ & 0.17 \\
\hline \multirow[t]{4}{*}{$1.62-1.83$} & 0.21 & & & $1.62-1.83$ & 0.21 & & \\
\hline & & $1.83-2.41$ & 0.58 & & & $1.83-1.98$ & 0.15 \\
\hline & & & & $1.98-2.00$ & 0.02 & $2.00-2.17$ & 0.17 \\
\hline & & & & $2.17-2.19$ & 0.02 & $2.19-2.41$ & 0.22 \\
\hline \multirow[t]{3}{*}{$2.41-2.84$} & 0.43 & & & $2.41-2.52$ & 0.11 & $2.52-2.54$ & 0.02 \\
\hline & & & & $2.54-2.84$ & 0.30 & & \\
\hline & & $2.84-2.91$ & 0.07 & & & $2.84-2.91$ & 0.07 \\
\hline $2.91-3.00$ & 0.09 & $3.00-3.10$ & 0.10 & $2.91-3.00$ & 0.09 & $3.00-3.10$ & 0.10 \\
\hline \multirow{4}{*}{$3.10-3.32$} & 0.22 & & & $3.10-3.32$ & 0.22 & & \\
\hline & & $3.32-3.76$ & 0.44 & & & $3.32-3.40$ & 0.08 \\
\hline & & & & $3.40-3.42$ & 0.02 & $3.42-3.57$ & 0.15 \\
\hline & & & & $3.57-3.59$ & 0.02 & $3.59-3.76$ & 0.17 \\
\hline $3.76-3.85$ & 0.09 & $3.85-3.97$ & 0.12 & $3.76-3.85$ & 0.09 & $3.85-3.97$ & 0.12 \\
\hline
\end{tabular}

vals with the durations of the new reverse polarity intervals being $0.15,0.17$ and $0.22 \mathrm{Ma}$. Clearly the original reverse polarity intervals have been split into shorter intervals of almost equal length, and

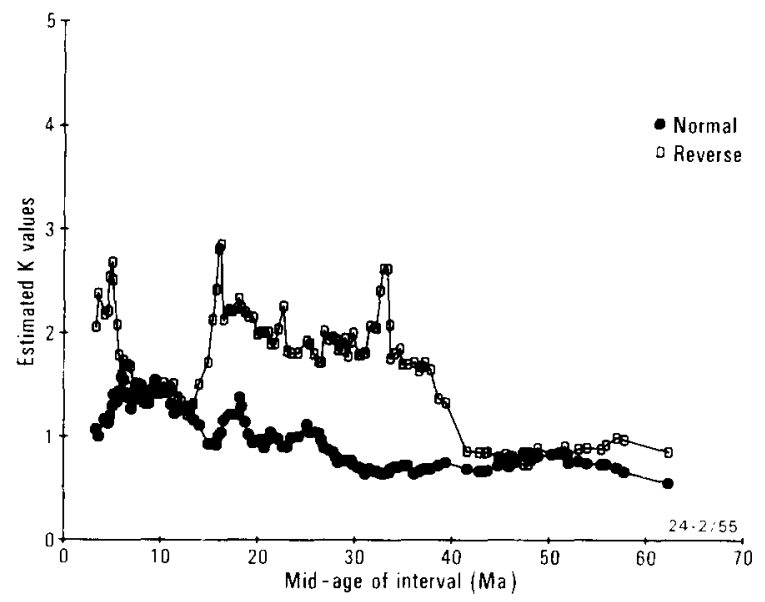

Fig. 4. Estimates of $k$ for the same data as Figure 3, except that the positioning of 2 of the added intervals has been adjusted. Sliding window as for Fig. 1. this has caused the sharp peak plotting at 3.5 Ma.

A small adjustment to the positioning of just two of the inserted short normal chrons (1.08-1.10 to $1.18-1.20$ and $1.43-1.45$ to $1.57-1.59$ ) produces the results plotted in Fig. 4. Certainly these results give no basis for rejecting the hypothesis of a common $k$ for the normal and reverse polarity sequences.

Overall, therefore, it is concluded that the data, either with or without the 57 very short chrons, are compatible with the normal and reverse sequences having a common $k$, and there is no need to call upon an asymmetry between the polarity states.

\section{Mean lengths of the intervals}

As noted by McFadden [7], the parameter $k$ in fact contains little information regarding the relative stabilities of the two polarities: the mean length, $\mu$ (or equivalently the reversal rate $\lambda$ ), contains much more information in this regard. 


\subsection{Analysis without the additional short events}

Estimates for $\mu$ for the LKC timescale are plotted in Fig. 5. Again the close similarity with the NLC timescale can be seen by comparing this figure with Fig. 3 of $M \& M$. A robust method was developed by McFadden [7] for testing the hypothesis that $\lambda_{\mathrm{r}}=\lambda_{\mathrm{n}}=\lambda$, where $\lambda_{\mathrm{r}}$ is the rate of reversals for the reverse polarity state and $\lambda_{n}$ is the rate of reversals for the normal polarity state. In Fig. 6 the fraction of time spent in the reverse polarity state is plotted using a sliding window of 25 intervals of each polarity. The inner dotted lines are the $95 \%$ prediction limits for the number of truly independent observations represented in the diagram (only 3 in this instance), and the outer dotted lines are the $95 \%$ prediction limits taking into account the fact that a sliding window has been used. Clearly the data give no reason to reject the hypothesis of a common reversal rate for the two polarity states.

\subsection{Analysis with the additional short events}

As with the estimation of $k$, the short events are all added in with a single duration of $20 \mathrm{kyr}$. Naturally, when dealing with mean lengths or rates of reversal, a choice of $20 \mathrm{kyr}$ is more extreme than either 30 or $40 \mathrm{kyr}$. Estimates for $\mu$ for the LKC timescale with the short events added are plotted in Fig. 7, and as expected the mean lengths are dragged down, particularly around 35 Ma. As before, the data are plotted in Fig. 8 in

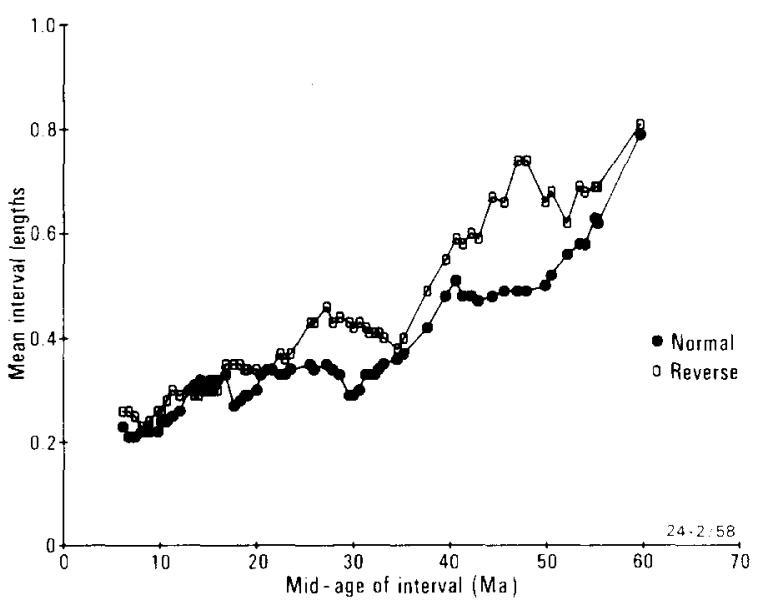

Fig. 5. Mean length of intervals for both polarities for the LKC timescale. Sliding window as for Fig. 1.

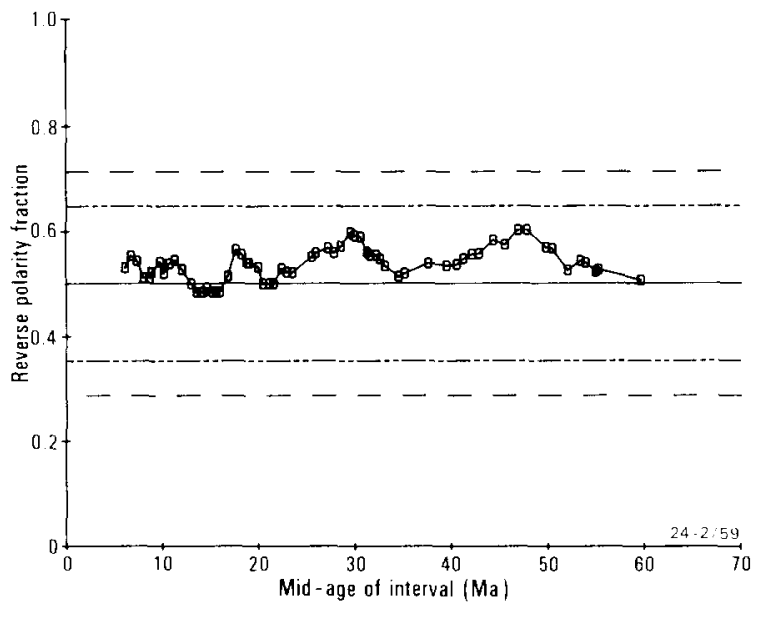

Fig. 6. Fraction of time spent in the reverse polarity state for the LKC timescale. Inner dotted lines are the $95 \%$ prediction limits for the number of independent observations and the outer dotted lines are the $95 \%$ prediction limits taking into account the use of a sliding window. Sliding window as for Fig. 1.

terms of the fraction of time spent in the reverse polarity state. As might be expected, near $35 \mathrm{Ma}$ the plot reaches the $95 \%$ prediction limits for the number of independent observations (inner dotted lines), but does not approach the $95 \%$ prediction limits that take into account the use of a sliding window (outer dotted lines).

Thus it is concluded that the data, either with or without the 57 very short chrons, are compati-

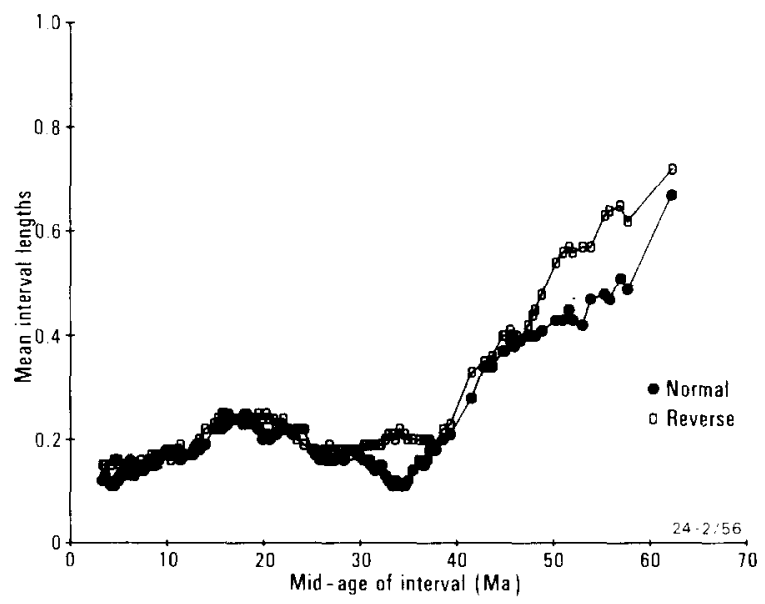

Fig. 7. Mean length of intervals for both polarities for the LKC timescale with the 57 short intervals added, each with a duration of 20 kyr. Sliding window as for Fig. 1. 


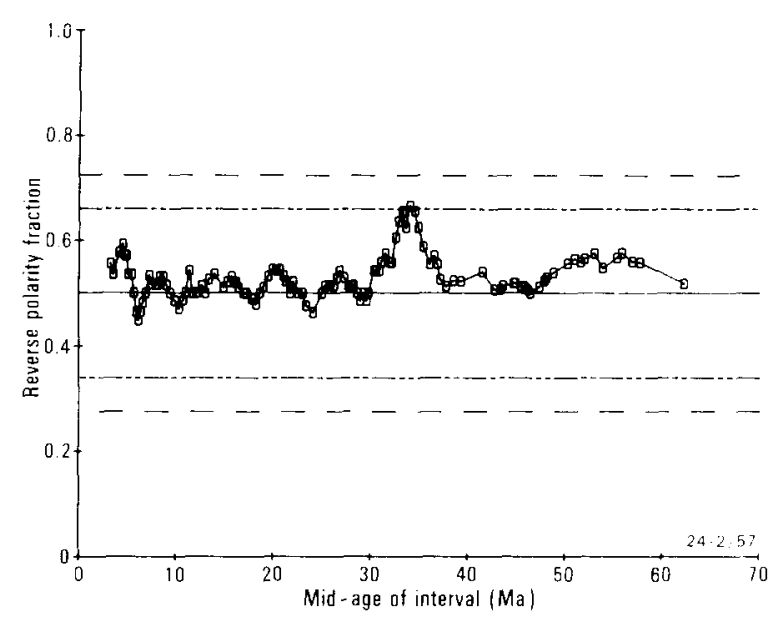

Fig. 8. Fraction of time spent in the reverse polarity state for the LKC timescale with the 57 short intervals added. Inner dotted lines are the $95 \%$ prediction limits for the number of independent observations and the outer dotted lines are the $95 \%$ prediction limits taking into account the use of a sliding window.

ble with the normal and reverse sequences having a common mean length or reversal rate.

\subsection{Rate of change of the reversal rate}

Using the NLC timescale $M \& M$ suggested that, to a first approximation, the reversal rate, $\lambda$, had changed linearly with time since the Cretaceous Normal Polarity Interval. This suggestion would also appear quite acceptable with the LKC timescale. However, it is clear from a comparison of Figs. 5 and 7 that the structure of the nonstationarity in $\lambda$ has been altered by the inclusion of the very short chrons. This is mainly due to the fact that of the 57 added chrons, only 7 occur prior to $38 \mathrm{Ma}$.

Hence, with the added chrons, the nonstationarity in the reversal rate no longer appears linear with time. However, it does still appear to change in a smooth fashion and to approach zero at the end of the Cretaceous Normal Polarity Interval. Consequently none of the geophysical interpretations of $\mathrm{M} \& \mathrm{M}$ is altered.

\section{Short events further back in the record}

As discussed earlier, there is now some evidence for 57 short reversal events in the Cenozoic not included in most reversal chronologies. In section 2 we suggest reasons why a disproportionately large number of these short events are of "normal polarity". Here we address the question of why so few of these short events (only 7 of the 57) have been identified in the $38-84 \mathrm{Ma}$ interval. Is this due to observational bias or degradation of the magnetic recorder, or is it a real geomagnetic phenomenon?

The best studied intervals are certainly those in the 0-38 Ma interval: anomalies 1 to 4 [22], anomalies 5 to 6 [11] and anomalies 12 to 13 [14]. In contrast, we note that with the few exceptions which produced the evidence for the seven pre-38 Ma short events [10,23], no comparable systematic global searches have been made for fine-scale magnetic anomaly features for the interval from about anomaly 15 to about anomaly 29 (ca. $38 \mathrm{Ma}$ to $66 \mathrm{Ma}$ ). Cande and Kristofferson [24] did, however, make a global survey of the Late Cretaceous interval from anomaly 29 to anomaly 34 (ca. $66 \mathrm{Ma}$ to $84 \mathrm{Ma}$ ). Thus, while some additional short polarity intervals may well have been missed in the 38-66 Ma interval through lack of detailed study, the comprehensive study by Cande and Kristofferson [24], which did not report any "tiny wiggles" in the older 66-84 Ma anomaly sequence, suggests that observational bias may not be that important in accounting for the relatively few short intervals in the reversal sequence prior to $38 \mathrm{Ma}$. We might also point out that magnetostratigraphic studies have not resulted in the documentation of very many additional short polarity intervals in the Paleocene, and virtually none in the Cretaceous Quiet Zone $[25,26]$. A systematic progressive loss of the high frequency component in the ocean floor magnetic recording has been offered as an explanation for the apparent decrease in reversal frequency with time [27]. A strong argument against this mechanism is provided by the existence of pre-M25 tiny wiggles [28], similar in frequency to those observed in the $0-38 \mathrm{Ma}$ interval, but recorded in ocean crust older than $156 \mathrm{Ma}$.

We therefore lean towards the remaining explanation for time variation in the occurrence of short events: that it approximates a real geomagnetic phenomenon. In addition to finding the alternative explanations inadequate, we would point to the observation that estimates of $k$ plotting 
back in time from about $38 \mathrm{Ma}$ (Fig. 3 or 4 ) tend to lie at around unity for both the normal and reverse polarity intervals. This suggests to us that there has not been a large number of short events missed in this interval. Instead, because the reversal rate was much lower, the "short" events were much longer and so most of them have been detected. Parenthetically, the much smaller number of "tiny wiggles" in the 38-84 Ma interval also argues against VRM-related mechanisms for their production in the $0-38 \mathrm{Ma}$ interval, because such mechanisms should produce tiny wiggles in older crust as well. Very detailed magnetostratigraphic and ocean magnetic surveys of the anomaly 15 to 29 sequence would provide a good test for confirming the geomagnetic significance we infer from the relative paucity of tiny wiggles in the 38-84 Ma interval.

\section{Periodicities in the reversal frequency}

In the past few years there have been several suggestions that there are periodicities within the reversal record [1,29-33]. However, the reality of these periodicities has been questioned [34,35]. The existence of a real periodic component in the reversal rate implies that the mean rate, $\lambda$, of the gamma process is given by:

$\lambda=\phi(t)+A_{1} \operatorname{Sin} \omega_{1} t+A_{2} \operatorname{Sin} \omega_{2} t+\ldots$

where $\phi(t)$ is the long-term variation already mentioned. As yet there has been no formal specification as to the value of any of the $A_{i}$. However, it is quite clear that if any genuine periodicities do exist, the amplitudes are very small.

A gamma process with a constant $\lambda$ produces intervals with varying lengths. If a filter designed to extract spectral information is applied to such a sequence it will indicate the presence of spectral components, even though each of the $A_{i}$ in equation (4) is zero and $\phi(t)$ is a constant. As may be expected, in each instance where a periodicity has been claimed, it is as a consequence of the application of a filter of some form to the reversal sequence. Naturally the situation is complicated further when $\phi(t)$ is not constant, as is the case with the geomagnetic reversal sequence.

Because of this, before concluding that a genuine periodicity exists, very careful testing must be performed to show that the observed periodic- ity is not merely a consequence of the random nature of the gamma process. Furthermore, if it is felt that a particular periodicity (equivalent to $\omega_{0}$ ) in the record is real, then because this periodicity has in fact been observed in the record, any testing must be conditioned upon the value $\omega_{0}$. Although some apparently compelling arguments have been put forward to substantiate the reality of periodicities (particularly by Stothers [33]), none of these has been backed up with testing conditional upon the observed periodicity. Consequently the question of genuine periodicities within the reversal record must, at this stage, be considered as unresolved.

\section{Conclusions}

Fifty-seven anomalies that appear on high-resolution magnetic anomaly records probably have multiple origins. Some probably reflect true missed polarity events, while others probably reflect changes in intensity in the record from other causes. Statistical analyses were carried out with two extreme assumptions. In one case, we assumed that all 57 anomalies reflect missed polarity events, while in the other case we assumed that none of the 57 anomalies represents a missed polarity event.

The analyses presented here show that the LKC timescale, either with or without the additional 57 short events, does not deny a time-averaged symmetry in the normal and reverse polarity states. The NLC timescale and the LKC timescale (without the additional 57 short events) are not truly independent because they were constructed from basically the same sets of data, and so, as expected, a comparison of their statistical properties shows a striking similarity. The addition of the 57 short events does alter the structure of the non-stationarity in the reversal rate, but each of the sequences (NLC, LKC, and LKC plus the 57 short events) is compatible with a smooth, and possibly monotonic, increase in the actual reversal rate from zero at the end of the Cretaceous Normal Polarity Interval to its present value in the region of 5 reversals per million years.

It is very satisfying that the same basic conclusions are drawn independent of whether one uses the NLC timescale, the LKC timescale, or the LKC timescale with the additional 57 short events. 
Thus it was not necessary to perform a rigorous determination of the validity of each of these short events before including it in the analysis. In effect this implies that the basic structure of the geomagnetic reversal history is now sufficiently well established that different scales and additional events only represent changes in detail. From this it may be concluded that the present geomagnetic polarity timescale for $0-160 \mathrm{Ma}$ is probably a very good representation of the geomagnetic reversal history.

\section{Acknowledgements}

R.T.M. appreciates partial support for this work from the National Science Foundation. D.V.K. appreciates support for this work from National Science Foundation Oceanography Division Grant OCE84-00805. LDGO Contribution \#4085. This paper is published with the permission of the Director, Bureau of Mineral Resources, Geology and Geophysics.

\section{References}

1 W. Lowrie and D.V. Kent, Geomagnetic reversal frequency since the Late Cretaceous, Earth Planet. Sci. Lett. 62, 305-313, 1983.

2 P.L. McFadden and R.T. Merrill, Lower mantle convection and geomagnetism, J. Geophys. Res. 89, 3354-3362, 1984.

3 R.T. Merrill, M.W. McElhinny and D.J. Stevenson, Evidence for long-term asymmetries in the earth's magnetic field and possible implications for dynamo theories, Phys. Earth Planet. Inter. 20, 75-82, 1979.

4 J.L. LaBrecque, D.V. Kent and S.C. Cande, Revised magnetic polarity time scale for Late Cretaceous and Cenozoic time, Geology 5, 330-335, 1977.

5 P.S. Naidu, Statistical structure of geomagnetic field reversals, J. Geophys. Res. 76, 2649-2662, 1971

6 G. Ness, S. Levi and R. Couch, Marine magnetic anomaly time scales for the Cenozoic and Late Cretaceous: a precis, critique and synthesis, Rev. Geophys. Space Phys. 18, $753-770,1980$

7 P.L. McFadden, Statistical tools for the analysis of geomagnetic reversal sequences, J. Geophys. Res. 89, 3363-3372, 1984.

8 J.D. Phillips, Time variation and asymmetry in the statistics of geomagnetic reversal sequences, I. Geophys. Res. 82 , $835-843,1977$.

9 D.V. Kent, Statistical structure of geomagnetic reversals. Nature 313, 15, 1985.

10 R.J. Blakely and A. Cox, Evidence for short geomagnetic polarity intervals in the Early Cenozoic, J. Geophys. Res. 77, 7065-7072, 1972.
11 R.J. Blakely, Geomagnetic reversals and crustal spreading rates during the Miocene, J. Geophys. Res. 79, 2979-2985. 1974.

12 A. Cox, Lengths of geomagnetic polarity intervals, J. Geophys. Res., 73, 3247-3260, 1968.

13 C.G.A. Harrison, What is the true rate of reversals of the Earth's magnetic field? Earth Sci. Planet. Lett. 6, 186-188, 1969.

14 S.C. Cande and J.L. LaBrecque, Behaviour of the earth's palaeomagnetic field from small scale marine magnetic anomalies, Nature 247, 26-28, 1974.

15 H.P. Johnson and R.T. Merrill, A direct test of the VineMatthews hypothesis, Earth Planet. Sci. Lett. 40, 263-269, 1978.

$16 \mathrm{H}$. Schouten and C.R. Denham, Modelling the oceanic source layer, in: Deep Sea Drilling Results in the Atlantic Ocean: Ocean Crust, M. Talwani, C.G. Harrison and D.E. Hayes, eds., Am. Geophys. Union, Maurice Ewing Ser., p. $151,1979$.

17 H.P. Johnson, Magnetisation of the oceanic crust, Rev. Geophys. Space Phys. 17, 215-226, 1979.

18 R.T. Merrill and M.W. McElhinny, The Earth's Magnetic Field: Its History, Origin and Planetary Perspective, 401 pp., Academic Press, New York, N.Y., 1983.

19 C.E. Barton, R.T. Merrill and M. Barbetti, Intensity of the earth's magnetic field over the last 10,000 years, Phys. Earth Planet. Inter. 20, 96-110, 1979.

20 W.E. Senanayake, M.W. McElhinny and P.L. McFadden, Comparison between the Thelliers' and Shaw's palaeointensity methods using basalts less than 5 million years old, $J$. Geomag. Geoelectr. 34, 141-161, 1982.

21 P.L. McFadden and R.T. Merrill, Geodynamo energy source constraints from palaeomagnetic data, Phys. Earth Planet. Inter. 43, 22-33, 1986

22 K.D. Klitgord, S.P. Huestis, J.D. Mudie and R.L. Parker, An analysis of near bottom magnetic anomalies: sea floor spreading and the magnetised layer, Geophys. J. R. Astron. Soc. 43, 387-424, 1975.

23 R. Schlich, Structure et age de l'ocean Indien occidental, Soc. Géol. Fr., Mem. Hors-Ser. 6, 103 pp., 1975.

24 S.C. Cande and Y. Kristofferson, Late Cretaceous magnetic anomalies in the North Atlantic, Earth Planet. Sci. Lett. 35 , 215-224, 1977.

25 W. Lowrie and W. Alvarez, Upper Cretaceous-Paleocene magnetic stratigraphy at Gubbio, Italy, III. Upper Cretaceous magnetic stratigraphy, Geol. Soc. Am. Bull. 88, 374- 377, 1977.

26 W. Lowrie, W. Alvarez, G. Napoleone, K. Perch-Nielsen, I. Premoli Silva and M. Toumarkine, Paleogene magnetic stratigraphy in Umbrian pelagic carbonate rocks: the Contessa sections, Gubbio, Geol. Soc. Am. Bull. 93, 414-432, 1982.

27 R.J. Blakely, An age-dependent, two-layer model for marine magnetic anomalies, in: The Geophysics of the Pacific Ocean Basin and Its Margin, G.H. Sutton, M.H. Manghnani, R. Moberly and E.U. McAgee, eds., Am. Geophys. Union, Geophys. Monogr. 19, 227-234, 1976.

28 S.C. Cande, R.L. Larson and J.L. LaBrecque, Magnetic lineations in the Pacific Jurassic Quiet Zone, Earth Planet. Sci. Lett. 41, 434-440, 1978. 
29 J.G. Negi and R.K. Tiwari, Matching long term periodicities of geomagnetic reversals and galactic motions of the solar system, Geophys. Res. Lett. 10, 713-716, 1983.

30 A. Mazaud, C. Laj, L. de Seze and K.L. Verosub, 15-Myr periodicity in the frequency of geomagnetic reversals since 100 Myr, Nature 304, 328-330, 1983.

31 D.M. Raup, Magnetic reversals and mass extinction, $\mathrm{Na}-$ ture 314, 341-343, 1985.

32 P.C. Pal and K.M. Creer, Geomagnetic reversal spurts and episodes of extraterrestrial catastrophism, Nature 320 , 148-150, 1986.

33 R.B. Stothers, Periodicity of the Earth's magnetic reversals, Nature, in press, 1986.

34 P.L. McFadden, 15-Myr periodicity in the frequency of geomagnetic reversals since $100 \mathrm{Myr}$, Nature 311, 396, 1984.

35 T.M. Lutz, The magnetic reversal record is not periodic, Nature 317, 404-407, 1985. 